\title{
Pupillometric analysis for assessment of gene therapy in Leber Congenital Amaurosis patients
}

\author{
Paolo Melillo ${ }^{1,2^{*}}$, Leandro Pecchia ${ }^{3}$, Francesco Testa ${ }^{1}$, Settimio Rossi ${ }^{1}$, Jean Bennett ${ }^{4,5}$ and Francesca Simonelli ${ }^{1}$
}

\author{
* Correspondence: \\ paolo.melillo@unina2.it \\ 'Department of Ophthalmology, \\ Second University of Naples, Naples, \\ Italy \\ ${ }^{2}$ Department of Electronics, \\ Computer Science and Systems, \\ University of Bologna, Bologna, Italy \\ Full list of author information is \\ available at the end of the article
}

\begin{abstract}
Background: Objective techniques to assess the amelioration of vision in patients with impaired visual function are needed to standardize efficacy assessment in gene therapy trials for ocular diseases. Pupillometry has been investigated in several diseases in order to provide objective information about the visual reflex pathway and has been adopted to quantify visual impairment in patients with Leber Congenital Amaurosis (LCA). In this paper, we describe detailed methods of pupillometric analysis and a case study on three Italian patients affected by Leber Congenital Amaurosis (LCA) involved in a gene therapy clinical trial at two follow-up time-points: 1 year and 3 years after therapy administration.
\end{abstract}

Methods: Pupillary light reflexes (PLR) were measured in patients who had received a unilateral subretinal injection in a clinical gene therapy trial. Pupil images were recorded simultaneously in both eyes with a commercial pupillometer and related software. A program was generated with MATLAB software in order to enable enhanced pupil detection with revision of the acquired images (correcting aberrations due to the inability of these severely visually impaired patients to fixate), and computation of the pupillometric parameters for each stimulus. Pupil detection was performed through Hough Transform and a non-parametric paired statistical test was adopted for comparison.

Results: The developed program provided correct pupil detection also for frames in which the pupil is not totally visible. Moreover, it provided an automatic computation of the pupillometric parameters for each stimulus and enabled semi-automatic revision of computerized detection, eliminating the need for the user to manually check frame by frame. With reference to the case study, the amplitude of pupillary constriction and the constriction velocity were increased in the right (treated eye) compared to the left (untreated) eye at both follow-up time-points, showing stability of the improved PLR in the treated eye.

Conclusions: Our method streamlined the pupillometric analyses and allowed rapid statistical analysis of a range of parameters associated with PLR. The results confirm that pupillometry is a useful objective measure for the assessment of therapeutic effect of gene therapy in patients with LCA.

Trial registration: ClinicalTrials.gov NCT00516477

\section{() Biomed Central}

\section{Background}

Leber congenital amaurosis (LCA) is a rare ocular disease, affecting around 1 in 81,000 people [1], and is one of the most severe forms of inherited retinal degeneration. LCA patients have severe loss of vision and abnormal eye movements (nystagmus) in early

(c) 2012 Melillo et al.; licensee BioMed Central Ltd. This is an Open Access article distributed under the terms of the Creative Commons Attribution License (http://creativecommons.org/licenses/by/2.0), which permits unrestricted use, distribution, and reproduction in any medium, provided the original work is properly cited. 
infancy and childhood. This disease has been associated with at least 15 different genes, and gene therapy for one of the forms, the congenital blindness disorder, LCA2, has been investigated recently in animals models and in humans [2-8].

The severe visual impairment in most patients affected by LCA, as well as other earlyonset retinal degenerations, is difficult to quantify with conventional clinical instrumentation [9]. In the past, there was no need to be exceedingly quantitative [10]. Recently, preclinical success in animal models of LCA and the development of several phase 1 clinical trials of gene therapy for LCA in humans had made it worthwhile to explore clinically feasible methods that can precisely quantify the visual function of these patients. Several techniques have been used in the first three independent clinical trials of LCA2 gene therapy, which initiated nearly contemporaneously in 2007 (NCT00481546[11], NCT00516477[8], NCT00643747 [12], ClinicalTrials.gov), in order to assess the improvement in visual function. Such techniques can be either subjective, that is, requiring an active response by the patients, or objective, that is, not requiring a voluntary response from the patients. As the gene therapy trials are open-label with the patients not blinded to the treatment, objective techniques have provided more reliable results. Until now, the objective ophthalmologic techniques applied in these studies have been exclusively electroretinogram and pupillometry. However, electroretinogram was unable to show the improvements achieved by gene therapy, as it was unrecordable both before and after treatment $[8,11,12]$. In contrast, pupillometry appeared to be a useful additional measure as it provides quantitative information in infants, in children and adults. Pupillometry consisted of the measurement of the light-induced contraction of the iris muscle due to the pupillary light reflex (PLR). The major signal input for PLR originates from rod and cone photoreceptors in the outer retina [13]. The accessibility of the iris for observation provides an easy, non-invasive, and non-contact method to explore visual function through the study of PLR. The adoption of pupillometry as a useful additional outcome measure in therapeutic trials of LCA was suggested in 2004 by Aleman who explored the feasibility of the technique to quantify the visual abnormalities in LCA patients [14]. Several studies investigated its utility for evaluation of improvement of light reflexes and asymmetry between the two eyes $[15,16]$. This evidence motivated the adoption of pupillometric analysis in the framework of the clinical gene therapy LCA trial registered as NCT00516477 in ClinicalTrials.gov [8]. The pupillometry was performed by using a commercial pupillometer and software. As the developed clinical protocol was unique, first a manual procedure was defined to perform the analysis but it was time-consuming. Successively, an ad hoc MATLAB package was developed in order to streamline the analysis and reduce the intervention of the operator, which may cause experimenter's bias.

In this paper, we propose and describe the most updated version of the pupillometric analysis method which has been explored in the framework of the clinical trial NCT00516477 for evaluation of pupillary reflexes in LCA2 patients undergoing gene therapy [8].

As a case study, we reported the results of the analysis of the three Italian patients involved in the clinical trial focusing on two post-treatment time-points at 1 year and 3 years respectively.

\section{Methods}

Case study

Three patients (further referred here as subjects 1, 2, and 3) involved in the clinical gene therapy LCA2 trial were analysed in this study: subject 1 is a 26 year old female, 
subject 2 is a 26 year old male, and subject 3 is a 19 year old female [8]. All patients were affected by LCA2 without other complications. None of the subjects took drugs affecting sympathetic or parasympathetic pupillary function. They had not past history of ocular operations, non-symmetrical pupil, misshapen pupil, or other conditions affecting pupillary reflexes. After informed consent and confirmation of trial eligibility, including legal blindness, the eye with worse visual function was selected for subretinal gene delivery through vector AAV2-hRPE65v2. In all the three patients, the right eye was injected. Subjects were evaluated before and after treatment as described in a previous report [8]. In this study, we focused on two post-treatment time-points: 12 months and 36 months.

\section{Pupillometric clinical protocol}

The stimulation protocol was modelled after the "swinging flashlight test", in order to identify relative afferent pupillary defects (rAPDs) [17]. Responses were measured after 40 minutes of dark adaptation by sequential stimulation in each eye with white light under low, medium and high intensity conditions (0.04, 0.4 and 10 lux, respectively) [17]. Pupillary responses to light were recorded with variants of the basic protocol, as described also elsewhere [6,7].

Two tests were performed:

- Test 1 - eight consecutive cycles consisting of a light stimulus presented for 0.2 seconds followed by a 1 second dark interval;

- Test 2 - six consecutive cycles consisting of a light stimulus presented for 1 second followed by a 0.6 second dark interval.

In both tests, the light stimulus was presented alternatively to the right and left eye and each test was repeated twice: the first time starting with the stimulation of the right eye, the second time starting with the stimulation of the left one. Each sequence of light stimulus was interleaved with a pause of at least 3 seconds in order to recover baseline pupil diameter. The tests were usually performed in the morning, after at least 8 hours of sleep.

\section{Pupillometric system}

Pupil responses were recorded simultaneously in both eyes with a Procyon P2000 pupillometer and PupilFit4 software (Monmouthshire, UK). Each eyepiece was equipped with infra-red diodes GaA1A's type SFH485 with peak emission at $880 \mathrm{~nm}$ to illuminate the pupil without stimulating the eye, and an infra-red sensitive camera that captured the video images at 25 frame/s, allowing the pupil diameter of both eyes to be measured every $40 \mathrm{~ms}$. Images were saved in bitmap format after real-time processing. Means, maximums and minimums of the pupil diameter for the entire test were computed by PupilFit4. Responses to individual stimuli were not computed. Therefore, a package of functions was developed in Matlab (The MathWorks Inc., Massachusetts, USA) to provide the following functionalities for each acquired frame:

- pupil detection;

- manual check/revision of pupil detection;

- elimination of unsuitable frames (e.g. due to blinking); 
- computation of pupillometric features for each stimulus;

- statistical analysis as described below.

The offline image processing algorithm determined the pupil diameter when the results of the PupilFit4 were not reliable, that is, when there were percentage variations in the pupil diameter from previous frames higher than a user-defined threshold (10\% default).

The algorithm consisted of four major steps:

- image thresholding - as the images were acquired under the same light condition, the darkness of the pupil is approximately the same for all the subjects (around 35 gray level), for that reason the threshold was set at the same value (40 gray level).

- hole filling - holes, mainly due to light reflexes, were filled using an algorithm based on morphological reconstruction [18].

- small object removing - all connected components (objects) that had fewer than a $\mathrm{N}$ pixels, were removed from the image. The number $\mathrm{N}$ was chosen in order to eliminate objects with a surface area less than $2 \mathrm{~mm}^{2}$, equivalent to a circle with a radius of $0.8 \mathrm{~mm}$.

- edge detection - morphological operations, such as dilatation and removing the interior pixel, were performed in order to leave only the boundary pixels, which should represent the pupillary border.

- pupil detection and diameter measurement - generalized Hough transform was implemented to detect the position and the diameter of the pupil, modelled as a circular shape.

Figure 1 shows the images obtained in the major algorithm steps until the best circle that fits the pupil is found.

A user-friendly Graphical User Interface was developed to check the results of the automatic pupil detection. It shows the image of the eye and the shape of the pupil detected by the off-line processing. If the user is satisfied by the automatic pupil detection, he/she just has to click the mouse, otherwise he/she can adjust the position and diameter of the detected shape by using the mouse. If the pupil could not be detected, not even manually, for instance due to blinking, the user could evaluate whether to accept a linear interpolation of the diameter between neighbor frames not affected by noise or, in extremis, to discard the stimulus.

According to the experience of previous studies $[6,7,19]$, pupillometric data were quantified through the following parameters:

-Baseline Diameter (BD) computed as the average of the pupil diameter $100 \mathrm{~ms}$ prior to the light stimulus;

-Minimum Amplitude (MA) defined as the lowest pupil diameter after a light stimulus; Response Amplitude (RA) computed as the difference between BD and MA;

-Percent Constriction (PC) defined as the ratio between MA and BD expressed as percentage;

- Response Time (RT) defined as the difference between the time corresponding to the minimum amplitude and the time corresponding to the stimulus;

-Constriction Velocity (CV) computed as the ratio between RA and RT. 


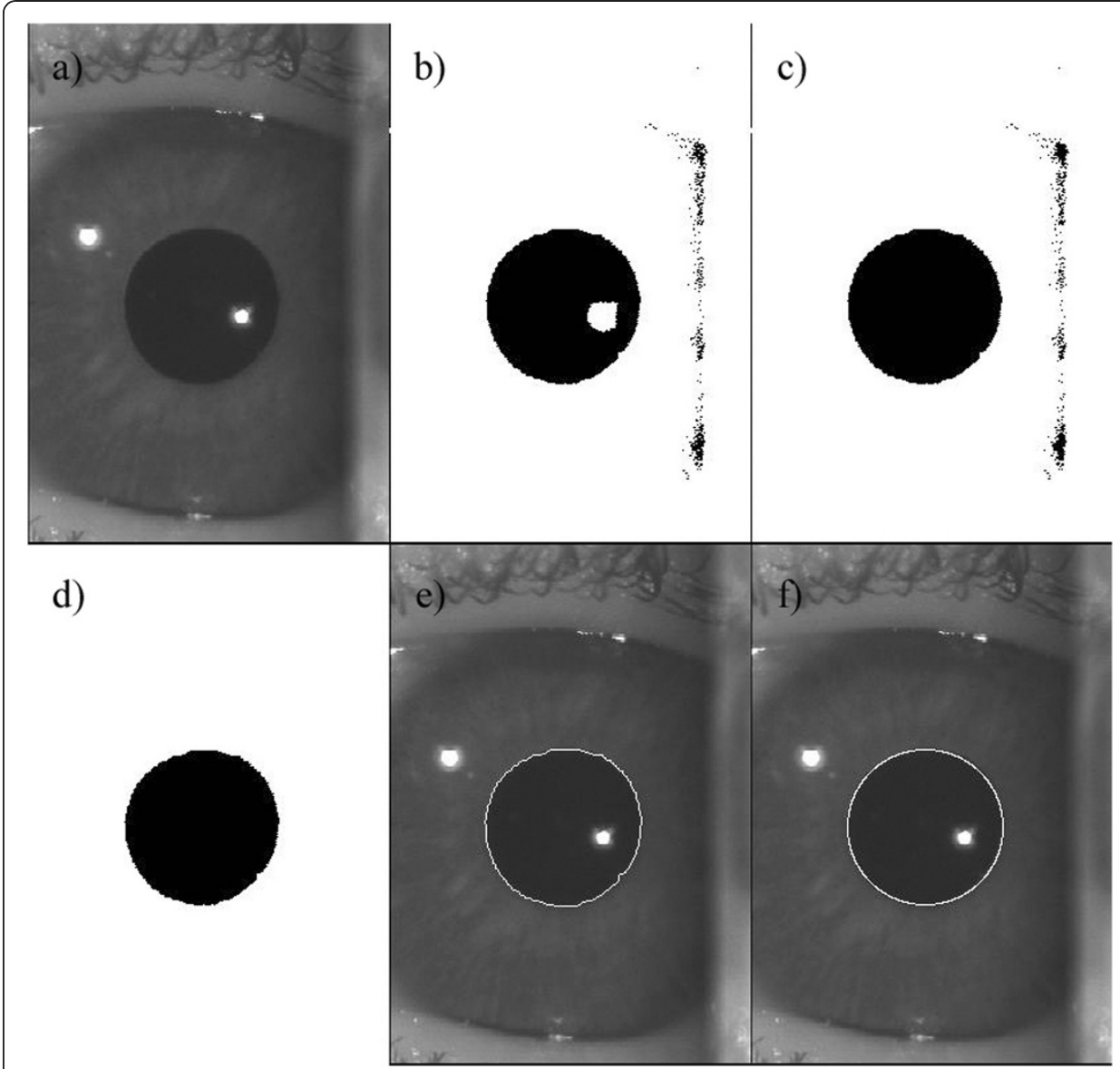

Figure 1 Description of the off-line pupil detection algorithm steps. (a) image acquired by the pupillometer; (b) image after the thresholding; (c) image after hole filling; (d) image after small object removing; (e) original image with the pupil border enhanced in white thanks to edge detection. (f) original image with the circle (in white) which fits the pupil border.

Figure 2 shows an example of pupil reflex and the definition of the parameters.

A non-parametric statistical paired test (Wilcoxon signed rank test) was performed to compare:

- the pupillometric parameters obtained when the right eye was stimulated with those obtained when the left eye was stimulated;

- the pupillometric parameters obtained at the first time-point (1 year after treatment) with those obtained at the second time-point (3 years after treatment).

\section{Results}

The developed MATLAB package provided an automatic pupil detection, which was correct also for frames in which the pupil was not totally visible (up to $30 \%$ of area not visible), for instance when the eyelashes obscured the pupil, as shown in Figure 3. When the automatic detection is not accurate, the user can manually revise the pupil detection.

The results related to the comparison between the two eyes are reported in Tables 1, 2 and 3 for each subject with respect to Test 1 . 


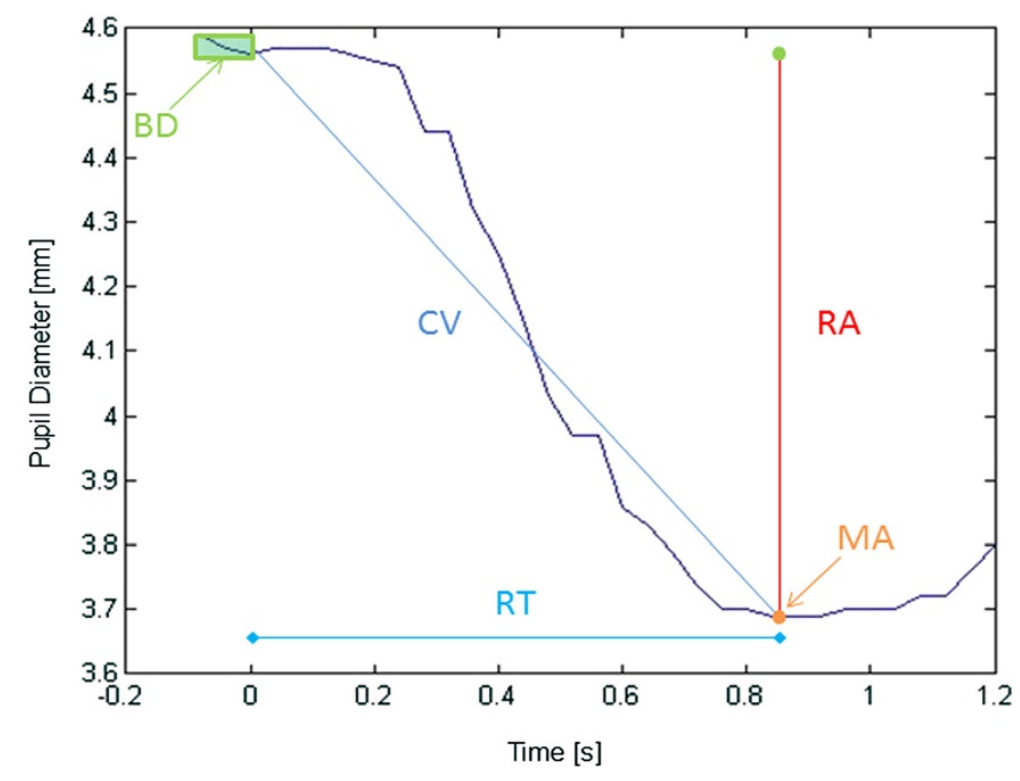

Figure 2 Pupil reflex of a subject involved in the study after a 0.2 second light stimulus triggered at zero second. The indicated parameters are computed as follows: BD, average of the pupil diameters $100 \mathrm{~ms}$ prior to the light stimulus (values to be averaged are in the green rectangular box): MA, the lowest pupil diameter after a light stimulus (represented by the orange point): RA, BD-MA (represented by the red segment); $R T$, $t_{\text {minimum amplitude- }} t_{\text {stimulus }}(t=$ time) (represented by the light blue segment): $C V, R A / R T$ (represented by the slope of the dark blue line).

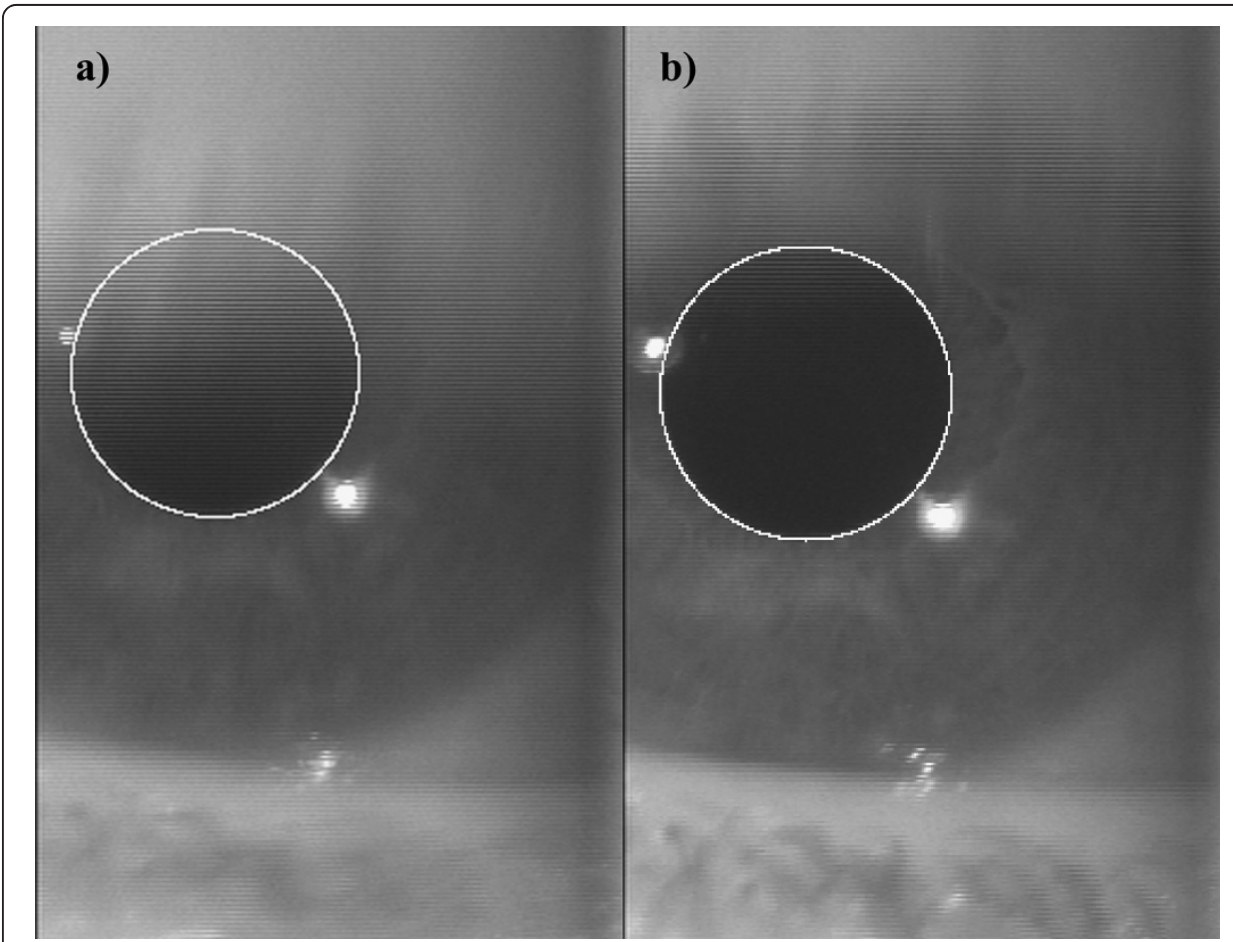

Figure 3 Example of pupil diameter detection when the eyelashes are over the pupil (a) and on the subsequent frame in which the pupil is completely visible (b). 
Table 1 Comparison of pupillometric parameters between the two eyes in subject 1 (Test 1)

\begin{tabular}{|c|c|c|c|c|c|c|c|c|c|c|c|c|c|c|}
\hline \multirow[t]{3}{*}{ Par. } & \multicolumn{7}{|c|}{1 year post-treatment } & \multicolumn{7}{|c|}{3 years post-treatment } \\
\hline & \multicolumn{3}{|c|}{ Right eye } & \multicolumn{3}{|c|}{ Left eye } & \multirow[b]{2}{*}{$\mathbf{P}$} & \multicolumn{3}{|c|}{ Right eye } & \multicolumn{3}{|c|}{ Left eye } & \multirow[b]{2}{*}{$\mathbf{P}$} \\
\hline & $\overline{\text { Med }}$ & $25^{\text {th }}$ & $75^{\text {th }}$ & Med & $25^{\text {th }}$ & $75^{\text {th }}$ & & $\overline{\text { Med }}$ & $25^{\text {th }}$ & $75^{\text {th }}$ & Med & $25^{\text {th }}$ & $75^{\text {th }}$ & \\
\hline $\mathrm{BD}$ & 4.67 & 4.30 & 4.98 & 4.47 & 3.89 & 4.69 & $<0.01$ & 4.17 & 3.75 & 4.60 & 4.14 & 3.60 & 4.54 & 0.21 \\
\hline MA & 4.35 & 3.78 & 4.57 & 4.37 & 4.03 & 4.70 & 0.02 & 4.07 & 3.52 & 4.34 & 4.06 & 3.54 & 4.39 & 0.69 \\
\hline BA & 0.37 & 0.28 & 0.52 & 0.02 & -0.09 & 0.06 & $<0.01$ & 0.20 & 0.04 & 0.33 & 0.04 & 0.00 & 0.14 & 0.01 \\
\hline $\mathrm{PC}$ & 0.92 & 0.89 & 0.94 & 1.00 & 0.98 & 1.02 & $<0.01$ & 0.96 & 0.92 & 0.99 & 0.99 & 0.97 & 1.00 & 0.01 \\
\hline & 0.98 & 0.84 & 1.04 & 0.34 & 0.20 & 0.70 & $<0.01$ & 0.90 & 0.48 & 1.02 & 0.26 & 0.22 & 1.04 & 0.10 \\
\hline 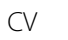 & 0.39 & 0.31 & 0.51 & 0.05 & -0.27 & 0.15 & $<0.01$ & 0.25 & 0.04 & 0.44 & 0.06 & 0.00 & 0.19 & 0.01 \\
\hline
\end{tabular}

Par: parameters.

Med: median.

$25^{\text {th }}: 25^{\text {th }}$ percentile (first quartile) $/ 75^{\text {th }}: 75^{\text {th }}$ percentile (third quartile).

P: $p$-value.

At 1 year follow-up, Response Amplitude (RA) and Constriction Velocity (CV) were higher when the injected (right) eye was stimulated compared to when the uninjected (left) eye was stimulated. These differences were statistically significant in all three subjects (with the exception of CV in patient 2). The asymmetry between left and right eye persisted through 3 years, the latest time-point tested, even if the differences of RA were statistically significant only in subjects 1 and 2 and the differences of CV were statistically significant only in subject 1 . In Test 2, RA and CV were higher when the injected (right) eye was stimulated instead of the uninjected (left) eye at both follow-up time-points, even if the differences were not statistically significant. Results of Test 2 are not reported in details as no statistical difference was found for any parameter.

The results of the comparison between the two follow-up time-points were reported in Tables 4, 5 and 6 for each subject. No significant difference in RA, PC, RT, and CV between 1-year and 3-year time-points was apparent when data from both eyes were considered (neither Test 1 nor Test 2). Significant differences were found in BD and MA for subjects 1 and 2 .

Table 2 Comparison of pupillometric parameters between the two eyes in subject 2 (Test 1)

\begin{tabular}{|c|c|c|c|c|c|c|c|c|c|c|c|c|c|c|}
\hline \multirow[t]{3}{*}{ Par. } & \multicolumn{7}{|c|}{1 year post-treatment } & \multicolumn{7}{|c|}{3 years post-treatment } \\
\hline & \multicolumn{3}{|c|}{ Right eye } & \multicolumn{3}{|c|}{ Left eye } & \multirow[b]{2}{*}{$P$} & \multicolumn{3}{|c|}{ Right eye } & \multicolumn{3}{|c|}{ Left eye } & \multirow[b]{2}{*}{$\mathbf{P}$} \\
\hline & Med & $25^{\text {th }}$ & $75^{\text {th }}$ & Med & $25^{\text {th }}$ & $75^{\text {th }}$ & & Med & $25^{\text {th }}$ & $75^{\text {th }}$ & Med & $25^{\text {th }}$ & $75^{\text {th }}$ & \\
\hline BD & 5.24 & 4.42 & 5.72 & 5.09 & 4.26 & 5.77 & 0.35 & 4.92 & 3.91 & 5.63 & 4.91 & 3.81 & 5.62 & 0.66 \\
\hline MA & 4.84 & 4.04 & 5.62 & 5.08 & 4.16 & 5.57 & 0.57 & 4.71 & 3.67 & 5.50 & 4.71 & 3.76 & 5.52 & 0.33 \\
\hline RA & 0.21 & 0.14 & 0.33 & 0.06 & 0.00 & 0.22 & 0.02 & 0.16 & 0.08 & 0.28 & 0.09 & 0.03 & 0.16 & 0.02 \\
\hline PC & 0.96 & 0.93 & 0.98 & 0.99 & 0.96 & 1.00 & 0.02 & 0.97 & 0.93 & 0.98 & 0.98 & 0.97 & 1.00 & 0.01 \\
\hline RT & 0.76 & 0.60 & 1.16 & 0.30 & 0.20 & 0.94 & 0.049 & 0.72 & 0.50 & 0.94 & 0.86 & 0.54 & 1.10 & 0.38 \\
\hline CV & 0.30 & 0.22 & 0.48 & 0.14 & -0.02 & 0.29 & 0.098 & 0.22 & 0.11 & 0.42 & 0.12 & 0.04 & 0.19 & 0.03 \\
\hline
\end{tabular}

Par: parameters.

Med: median.

$25^{\text {th }}: 25^{\text {th }}$ percentile (first quartile) $/ 75^{\text {th }}: 75^{\text {th }}$ percentile (third quartile).

P: p-value. 
Table 3 Comparison of pupillometric parameters between the two eyes in subject 3 (Test 1)

\begin{tabular}{|c|c|c|c|c|c|c|c|c|c|c|c|c|c|c|}
\hline \multirow[t]{3}{*}{ Par. } & \multicolumn{7}{|c|}{1 year post-treatment } & \multicolumn{7}{|c|}{3 years post-treatment } \\
\hline & \multicolumn{3}{|c|}{ Right eye } & \multicolumn{3}{|c|}{ Left eye } & \multirow[b]{2}{*}{$\mathbf{P}$} & \multicolumn{3}{|c|}{ Right eye } & \multicolumn{3}{|c|}{ Left eye } & \multirow[b]{2}{*}{$\mathbf{P}$} \\
\hline & Med & $25^{\text {th }}$ & $75^{\text {th }}$ & Med & $25^{\text {th }}$ & $75^{\text {th }}$ & & Med & $25^{\text {th }}$ & $75^{\text {th }}$ & Med & $25^{\text {th }}$ & $75^{\text {th }}$ & \\
\hline $\mathrm{BD}$ & 4.00 & 3.84 & 4.03 & 3.75 & 3.56 & 4.00 & 0.07 & 3.85 & 3.64 & 4.42 & 3.74 & 3.61 & 4.32 & 0.88 \\
\hline MA & 3.52 & 3.38 & 3.63 & 3.70 & 3.47 & 3.78 & 0.03 & 3.65 & 3.47 & 4.01 & 3.75 & 3.44 & 4.14 & 0.64 \\
\hline RA & 0.39 & 0.29 & 0.51 & 0.05 & -0.03 & 0.23 & $<0.01$ & 0.20 & 0.14 & 0.33 & 0.08 & -0.02 & 0.23 & 0.10 \\
\hline$P C$ & 0.90 & 0.87 & 0.92 & 0.98 & 0.94 & 1.01 & $<0.01$ & 0.94 & 0.92 & 0.96 & 0.98 & 0.94 & 1.01 & 0.10 \\
\hline $\mathrm{RT}$ & 0.72 & 0.56 & 0.94 & 0.52 & 0.20 & 0.68 & $<0.01$ & 0.66 & 0.52 & 0.90 & 0.66 & 0.60 & 0.80 & 0.78 \\
\hline CV & 0.63 & 0.47 & 0.69 & 0.12 & -0.09 & 0.34 & $<0.01$ & 0.33 & 0.21 & 0.51 & 0.13 & -0.06 & 0.36 & 0.11 \\
\hline
\end{tabular}

Moreover, the analysis was performed also by distinguishing PLR according to the light intensity stimulus. The asymmetry between the two eyes with respect to RA, was statically significant in subject 1 at all three intensity levels, in subject 2 only at the highest one, in subject 3 only at the lower one.

\section{Discussion}

In this study, we describe the application of pupillometric analysis for evaluation of pupillary reflexes in patients undergoing gene therapy. This is clinically relevant because the pupillometry appeared to be the only objective ophthalmologic test able to detect an amelioration of visual function in treated LCA patients. The results showed that the patients had a higher PLR when the treated eye was stimulated rather than when the control one was stimulated. These findings are especially noteworthy as before treatment the treated eye had the worst responses [8]. The absence of statistical differences between the two selected time-points suggested stability over time of therapeutic effect mediated by gene delivery. These results were consistent with the improvement and the stability over time of other clinical parameters such as best corrected visual acuity [8]. As expected according to Kawasaki [20], Test 1, based on

Table 4 Comparison of pupillometric parameters between the two follow-up time-points in subject 1 (Both Tests)

\begin{tabular}{|c|c|c|c|c|c|c|c|c|c|c|c|c|c|c|}
\hline \multirow[t]{3}{*}{ Par. } & \multicolumn{7}{|c|}{ Test 1} & \multicolumn{7}{|c|}{ Test 2} \\
\hline & \multicolumn{3}{|c|}{1 year time-point } & \multicolumn{3}{|c|}{3 year time-point } & \multirow[b]{2}{*}{$\mathbf{P}$} & \multicolumn{3}{|c|}{1 year time-point } & \multicolumn{3}{|c|}{3 year time-point } & \multirow[b]{2}{*}{$\mathbf{P}$} \\
\hline & Med & $25^{\text {th }}$ & $75^{\text {th }}$ & Med & $25^{\text {th }}$ & $75^{\text {th }}$ & & Med & $25^{\text {th }}$ & $75^{\text {th }}$ & Med & $25^{\text {th }}$ & $75^{\text {th }}$ & \\
\hline $\mathrm{BD}$ & 4.56 & 4.20 & 4.77 & 4.14 & 3.71 & 4.59 & $<0.01$ & 4.56 & 4.00 & 4.81 & 4.03 & 3.93 & 4.24 & $<0.01$ \\
\hline MA & 4.37 & 3.91 & 4.60 & 4.06 & 3.52 & 4.38 & $<0.01$ & 4.22 & 3.67 & 4.73 & 3.95 & 3.70 & 4.06 & $<0.01$ \\
\hline RA & 0.16 & 0.01 & 0.39 & 0.06 & 0.01 & 0.25 & 0.06 & 0.16 & 0.06 & 0.29 & 0.16 & 0.05 & 0.28 & 0.26 \\
\hline$P C$ & 0.96 & 0.92 & 1.00 & 0.99 & 0.94 & 1.00 & 0.11 & 0.97 & 0.93 & 0.99 & 0.96 & 0.93 & 0.99 & 0.42 \\
\hline$-R T$ & 0.74 & 0.28 & 1.00 & 0.80 & 0.24 & 1.04 & 0.77 & 0.94 & 0.68 & 1.22 & 1.00 & 0.48 & 1.24 & 0.77 \\
\hline CV & 0.26 & 0.02 & 0.42 & 0.13 & 0.04 & 0.30 & 0.30 & 0.14 & 0.07 & 0.33 & 0.18 & 0.05 & 0.31 & 0.48 \\
\hline
\end{tabular}

Par: parameters.

Med: median.

$25^{\text {th }}: 25^{\text {th }}$ percentile (first quartile) $/ 75^{\text {th }}: 75^{\text {th }}$ percentile (third quartile).

P: p-value. 
Table 5 Comparison of pupillometric parameters between the two follow-up time-points in subject 2 (Both Tests)

\begin{tabular}{|c|c|c|c|c|c|c|c|c|c|c|c|c|c|c|}
\hline \multirow[t]{3}{*}{ Par. } & \multicolumn{7}{|c|}{ Test 1} & \multicolumn{7}{|c|}{ Test 2} \\
\hline & \multicolumn{3}{|c|}{1 year time-point } & \multicolumn{3}{|c|}{3 year time-point } & \multirow[b]{2}{*}{$\mathbf{P}$} & \multicolumn{3}{|c|}{1 year time-point } & \multicolumn{3}{|c|}{3 year time-point } & \multirow[b]{2}{*}{$\mathbf{P}$} \\
\hline & Med & $25^{\text {th }}$ & $75^{\text {th }}$ & Med & $25^{\text {th }}$ & $75^{\text {th }}$ & & Med & $25^{\text {th }}$ & $75^{\text {th }}$ & Med & $25^{\text {th }}$ & $75^{\text {th }}$ & \\
\hline BD & 5.15 & 4.30 & 5.77 & 4.92 & 3.82 & 5.62 & $<0.01$ & 4.99 & 4.26 & 5.23 & 4.67 & 4.19 & 5.13 & 0.55 \\
\hline MA & 5.05 & 4.11 & 5.57 & 4.71 & 3.71 & 5.50 & $<0.01$ & 4.65 & 4.05 & 4.97 & 4.36 & 3.88 & 4.90 & 0.48 \\
\hline & 0.17 & 0.06 & 0.28 & 0.11 & 0.04 & 0.22 & 0.24 & 0.24 & 0.05 & 0.34 & 0.24 & 0.05 & 0.43 & 0.53 \\
\hline & 0.97 & 0.95 & 0.99 & 0.97 & 0.96 & 0.99 & 0.68 & 0.95 & 0.93 & 0.99 & 0.95 & 0.90 & 0.99 & 0.54 \\
\hline & 0.62 & 0.20 & 1.06 & 0.72 & 0.52 & 1.08 & 0.30 & 1.04 & 0.56 & 1.50 & 1.10 & 0.70 & 1.34 & 0.80 \\
\hline$c$ & 0.26 & 0.13 & 0.34 & 0.15 & 0.07 & 0.26 & 0.38 & 0.20 & 0.09 & 0.34 & 0.21 & 0.05 & 0.44 & 0.89 \\
\hline
\end{tabular}

Par: parameters.

Med: median.

$25^{\text {th }}: 25^{\text {th }}$ percentile (first quartile) $/ 75^{\text {th }}: 75^{\text {th }}$ percentile (third quartile).

P: p-value.

short stimulus cycles and dark intervals, provided significant results compared to Test 2. The choice of presenting different intensity light stimulus was motivated by the findings by Bergamin [15], who concluded that the ability to detect asymmetry between the two eyes was best obtained by testing over a range of light intensity. For instance, if only the middle light intensity level had been adopted in this study, no significant differences would have been found in subjects 2 and 3. Moreover, results presented in this paper confirm the usefulness of pupillometry to assess effect of intervention in LCA patients.

Pupillometry has been widely investigated in patients with diabetes to provide information about autonomic neuropathy [21-24], showing that the baseline pupil size, diameter and time parameters during the contraction phase are mainly under sympathetic control while both sympathetic and para-sympathetic systems are active during the recovery phase. Since the selected patients showed no evidence of autonomic dysfunction, and the aim of the current study was not to show relationship between PLR parameters and autonomous nervous system (ANS), we focused on parameters derived from the contraction phase of the pupil reflex which, according to the findings by Bergamin [15], are more useful than those extracted from the dilatation phase to show asymmetry

Table 6 Comparison of pupillometric parameters between the two follow-up time-points in subject 3 (Both Tests)

\begin{tabular}{|c|c|c|c|c|c|c|c|c|c|c|c|c|c|c|}
\hline \multirow[t]{3}{*}{ Par. } & \multicolumn{7}{|c|}{ Test 1} & \multicolumn{7}{|c|}{ Test 2} \\
\hline & \multicolumn{3}{|c|}{1 year time-point } & \multicolumn{3}{|c|}{3 year time-point } & \multirow[b]{2}{*}{$\mathbf{P}$} & \multicolumn{3}{|c|}{1 year time-point } & \multicolumn{3}{|c|}{3 year time-point } & \multirow[b]{2}{*}{$\mathbf{P}$} \\
\hline & Med & $25^{\text {th }}$ & $75^{\text {th }}$ & Med & $25^{\text {th }}$ & $75^{\text {th }}$ & & Med & $25^{\text {th }}$ & $75^{\text {th }}$ & Med & $25^{\text {th }}$ & $75^{\text {th }}$ & \\
\hline $\mathrm{BD}$ & 3.98 & 3.69 & 4.02 & 3.79 & 3.64 & 4.35 & 0.66 & 3.65 & 3.41 & 4.01 & 3.97 & 3.39 & 4.22 & 0.32 \\
\hline MA & 3.55 & 3.40 & 3.72 & 3.67 & 3.47 & 4.08 & 0.08 & 3.38 & 3.09 & 3.62 & 3.49 & 3.03 & 3.96 & 0.03 \\
\hline RA & 0.23 & 0.05 & 0.46 & 0.16 & 0.03 & 0.24 & 0.15 & 0.32 & 0.12 & 0.62 & 0.30 & 0.19 & 0.44 & 0.57 \\
\hline PC & 0.94 & 0.89 & 0.98 & 0.96 & 0.94 & 0.99 & 0.12 & 0.91 & 0.84 & 0.96 & 0.92 & 0.89 & 0.95 & 0.57 \\
\hline RT & 0.62 & 0.48 & 0.82 & 0.66 & 0.58 & 0.84 & 0.24 & 0.90 & 0.66 & 1.18 & 0.98 & 0.64 & 1.12 & 0.96 \\
\hline CV & 0.38 & 0.10 & 0.65 & 0.22 & 0.04 & 0.46 & 0.11 & 0.33 & 0.13 & 0.66 & 0.32 & 0.22 & 0.42 & 0.67 \\
\hline
\end{tabular}

Par: parameters.

Med: median.

$25^{\text {th }}: 25^{\text {th }}$ percentile (first quartile) $/ 75^{\text {th }}: 75^{\text {th }}$ percentile (third quartile).

P: p-value. 
between the two eyes. In particular, RA appears to be the most useful parameter to show this asymmetry, consistently with the results of previous reports in the framework of the same clinical trial [6-8]. Obviously, PC, which is the ratio of RA and BD, provides the same information as RA. In contrast, in our patients $\mathrm{BD}$ appeared to be insensitive to measure visual impairment, coherently with the findings by Aleman [10], who observed near-normal pupil diameters under dark-adapted conditions in a cohort of 18 patients with LCA, compared to 8 healthy subjects.

The comparison of our results with the findings of other clinical gene therapy trial using pupillometric analysis [25] is limited by the differences in the adopted protocol [26]. Cideciyan [25] computed RA as the difference between BD and the pupil diameter at a fixed time after the onset of the stimulus and derived the so-called luminance-response functions from RA to stimulus of increasing intensities (over a $\sim 9 \log$ unit range). Cideciyan [25] reported that two of the three patients showed a shift of their luminance-response functions to lower stimulus intensities in their injected eye, signifying a better sensitivity, while there was no change in the control eyes in the three patients nor in the injected eye of a third patient. We underline that, in the current study and in the related clinical trial, a different approach was adopted based on binocular pupillometry method designed to detect relative afferent pupillary defects [17]. This method enabled to show significant improvement in PLR in all the patients after treatment, as previously reported [6-8]. Although the underlined differences, in both trials, transient/dynamic (as opposed to steady state/static) PLR elicited by short duration stimuli was adopted as suggested by Aleman [10, 14]. The results of both the trials confirm the feasibility and usefulness of dynamic pupillometry as an objective and non-invasive measure of the visual functionality in LCA patients, as also concluded by a recent conference abstract by Kawasaki [27].

As regards software implementation, the developed MATLAB package improved the on-line pupil detection and automatically computed the pupillometric parameters for each stimulus. We believe that a check by the user is required in this field, as it would be improper that a result derived from an incorrect automatic detection might affect the decision whether or not an expensive therapy should be further investigated or transferred to clinical practice. A manual standardized protocol had been developed to check the results of the online pupil detection but the procedure required about 60 minutes for each pupillometric time-series. For that reason, a Grafical User Interface was developed to enable a semi-automatic revision of computerized detection, eliminating the need of this time-consuming procedure in which the user should manually check frame by frame using other imaging software. We tested that the same results (particularly, in terms of frames identified because of inaccurate online pupil detection) were achieved but in about 5 minutes (versus about 60 minutes of the manual procedure) for each pupillometric time-series.

Other objective techniques, such as fMRI, have been investigated to provide objective information about the improvement in retinal and visual function of LCA2 patients treated by gene therapy [5]. fMRI has been shown to give useful information about visual cortex activity but it is a much more expensive technology and is not suitable for all patients, as some patients were unable (or not willing) to undergo MRI because of a medical contra-indication (for example, a prosthesis), claustrophobia, non-collaboration. 
This study presented some limitations, which are described in the following paragraphs. The implemented algorithm is designed to improve the real-time processing results provided by PupilFil4, and is not intended to be a method for real-time pupil measurement, such as those proposed by Zhu [28] or by Iskander [29]. The number of pupillometric parameters to be computed could be expanded, for instance the implementation of iris detection could be a further development in order to compute parameters based on the pupil-iris radius ratio as introduced by Fotiou [30]. However, the choice of the parameters was driven by the unique situation of severely impaired patients, who were legally blind before treatment. Another limitation is the small sample size, but it is typical of a phase 1 clinical trial in this field, as it is very hard to recruit a large number of patients with the selected mutation since LCA2 is a rare disease. Five Italian patients were recruited thanks to the national relevance of the ophthalmologic clinic in Naples, and, at the moment, data of long-term follow-up are available for these three patients.

An interesting further development could extend to using pupillometric data as a measure of the ANS activity, for instance in conjunction with the Heart Rate Variability, another non-invasive marker of the ANS activity, widely investigated by the authors (PM and LP) both in healthy subjects [31] and in patients [32-34].

\section{Conclusions}

In conclusion, the results shown in this paper confirm that pupillometry is a useful objective measure to assess the effect of gene therapy in LCA patients. The developed program streamlined the analyses and allowed rapid statistical analysis of a range of parameters associated with PLR. The methods could be suitable in randomized clinical trials in order to assess the efficacy of gene therapy in LCA patients with no/low risk of patients' and experimenters' bias. With reference to the case study, the pupillometric analysis provided further support for the persistence over the time (3 years) of a significant improvement of retinal/visual function in the treated eyes.

Competing interests

The authors declare that they have no competing interests.

\section{Authors' contributions}

PM conceived the study and developed the method for off-line processing and data analysis, LP revised them. JB provided the set-up of the hardware for the pupillometry measurement. FT, SR, JB and FS designed the clinical protocol. FT performed the pupillometric test and acted as user of the MATLAB software. PM drafted the manuscript, LP, FT, SR and JB participated to drafting of the manuscript. FT, SR and FS recruited the subjects. All authors read, reviewed and approved the final manuscript.

\section{Acknowledgements}

This work was supported by a Telethon grant (Naples, Italy; GGP07180 to FS). PM was supported by a scholarship in the framework of the same grant: "A safety and efficacy study in subjects with Leber Congenital Amaurosis (LCA) using Adeno-Associated Viral Vector to deliver the gene for Human RPE 65 to the Retinal Pigment Epithelium (RPE) [AAV2hRPE65v2-301]: treatment and follow up of 3 Italian patients". LP acknowledges support of this work through the MATCH Programme (EPSRC Grant EP/F063822/1) although the views expressed are entirely his own. All the authors thank Carmela Acerra for her linguistic revision and the team at the Center for Cellular and Molecular Therapeutics at the Children's Hospital of Philadelphia for designing the initial studies, providing the pupillometer, obtaining regulatory approvals in the USA, and directing the conduct of the Phase I clinical trial for LCA2.

\section{Author details}

${ }^{1}$ Department of Ophthalmology, Second University of Naples, Naples, Italy. ${ }^{2}$ Department of Electronics, Computer Science and Systems, University of Bologna, Bologna, Italy. ${ }^{3}$ Department of Electrical \& Electronic Engineering, University of Nottingham, Nottingham, United Kingdom. ${ }^{4}$ Center for Cellular and Molecular Therapeutics, Children's Hospital of Philadelphia (CHOP), Philadelphia, PA, USA. ${ }^{5}$ F.M. Kirby Center for Molecular Ophthalmology, University of Pennsylvania, Philadelphia, PA, USA. 


\section{References}

1. Stone EM: Leber congenital amaurosis - a model for efficient genetic testing of heterogeneous disorders: LXIV Edward Jackson memorial lecture. Am J Ophthalmol 2007, 144:791-811.

2. Banin E, Bandah-Rozenfeld D, Obolensky A, Cideciyan AV, Aleman TS, Marks-Ohana D, Sela M, Boye S, Sumaroka A, Roman AJ, et al: Molecular anthropology meets genetic medicine to treat blindness in the North African Jewish population: human gene therapy initiated in Israel. Hum Gene Ther 2010, 21:1749-1757.

3. Hauswirth WW, Aleman TS, Kaushal S, Cideciyan AV, Schwartz SB, Wang L, Conlon TJ, Boye SL, Flotte TR, Byrne BJ, Jacobson SG: Treatment of leber congenital amaurosis due to RPE65 mutations by ocular subretinal injection of adeno-associated virus gene vector: short-term results of a phase I trial. Hum Gene Ther 2008, 19:979-990.

4. Artur VC: Leber congenital amaurosis due to RPE65 mutations and its treatment with gene therapy. Prog Retin Eye Res 2010, 29:398-427.

5. Bennett J, Ashtari M, Wellman J, Marshall KA, Cyckowski LL, Chung DC, McCague S, Pierce EA, Chen Y, Bennicelli $J \mathrm{~L}$, et al: AAV2 gene therapy readministration in three adults with congenital blindness. Sci Trans/ Med 2012, 4:120ra115.

6. Maguire AM, High KA, Auricchio A, Wright JF, Pierce EA, Testa F, Mingozzi F, Bennicelli JL, Ying GS, Rossi S, et al: Age-dependent effects of RPE65 gene therapy for Leber's congenital amaurosis: a phase 1 dose-escalation trial. Lancet 2009, 374:1597-1605.

7. Simonelli F, Maguire AM, Testa F, Pierce EA, Mingozzi F, Bennicelli JL, Rossi S, Marshall K, Banfi S, Surace EM, et al: Gene therapy for Leber's congenital amaurosis is safe and effective through 1.5 years after vector administration. Mol Ther 2010, 18:643-650.

8. Maguire AM, Simonelli F, Pierce EA, Pugh EN Jr, Mingozzi F, Bennicelli J, Banfi S, Marshall KA, Testa F, Surace EM, et al: Safety and efficacy of gene transfer for Leber's congenital amaurosis. N Engl J Med 2008, 358:2240-2248.

9. Fazzi E, Signorini SG, Scelsa B, Bova SM, Lanzi G: Leber's congenital amaurosis: an update. Eur J Paediatr Neurol $2003,7: 13-22$

10. Aleman TS, Jacobson SG, Chico JD, Scott ML, Cheung AY, Windsor EA, Furushima M, Redmond TM, Bennett J, Palczewski K, Cideciyan AV: Impairment of the transient pupillary light reflex in Rpe65(-/-) mice and humans with leber congenital amaurosis. Invest Ophthalmol Vis Sci 2004, 45:1259-1271.

11. Cideciyan AV, Hauswirth WW, Aleman TS, Kaushal S, Schwartz SB, Boye SL, Windsor EA, Conlon TJ, Sumaroka A, Pang JJ, et al: Human RPE65 gene therapy for Leber congenital amaurosis: persistence of early visual improvements and safety at 1 year. Hum Gene Ther 2009, 20:999-1004.

12. Bainbridge JW, Smith AJ, Barker SS, Robbie S, Henderson R, Balaggan K, Viswanathan A, Holder GE, Stockman A, Tyler N, et al: Effect of gene therapy on visual function in Leber's congenital amaurosis. N Engl I Med 2008, 358:2231-2239.

13. Foster RG: Keeping an eye on the time: the Cogan lecture. Invest Ophthalmol Vis Sci 2002, 43:1286-1298.

14. Aleman T, Scott M, Chen R, Chico J, Cheung A, Van Hooser J, Redmond T, Palczewski K, Jacobson S, Cideciyan A: Impairment of the pupillary light reflex in Rpe65-/- mice and patients with leber congenital amaurosis. Invest Ophthalmol Vis Sci 2002, 43:3490.

15. Bergamin O, Zimmerman MB, Kardon RH: Pupil light reflex in normal and diseased eyes: diagnosis of visual dysfunction using waveform partitioning. Ophthalmology 2003, 110:106-114.

16. Kardon R, Kawasaki A, Miller NR: Origin of the relative afferent pupillary defect in optic tract lesions. Ophthalmology 2006, 113:1345-1353.

17. Volpe NJ, Dadvand L, Kim SK, Maguire MG, Ying G-S, Moster ML, Galetta SL: Computerized binocular pupillography of the swinging flashlight test detects afferent pupillary defects. Curr Eye Res 2009, 34:606-613.

18. Soille P: Morphological image analysis: principles and applications. Berlin: Springer; 1999.

19. Canver MC, Canver AC, Revere KE, Amado D, Bennett J, Chung DC: Algorithm for pupillometric data analysis. In Bioengineering conference, proceedings of the 2010 IEEE 36th Annual Northeast; 26-28 March 2010. Edited by Samul K. New York: IEEE; 2010:1-2.

20. Kawasaki A, Moore P, Kardon RH: Variability of the relative afferent pupillary defect. Am J Ophthalmol 1995, 120:622-633.

21. Dutsch $M$, Marthol $H$, Michelson $G$, Neundorfer B, Hilz MJ: Pupillography refines the diagnosis of diabetic autonomic neuropathy. J Neurol Sci 2004, 222:75-81.

22. Ferrari G, Marques J, Gandhi R, Heller S, Schneider F, Tesfaye S, Gamba H: Using dynamic pupillometry as a simple screening tool to detect autonomic neuropathy in patients with diabetes: a pilot study. Biomed Eng Online 2010, 9:26.

23. Smith SA, Dewhirst RR: A simple diagnostic test for pupillary abnormality in diabetic autonomic neuropathy. Diabet Med 1986, 3:38-41.

24. Smith SA, Smith SE: Reduced pupillary light reflexes in diabetic autonomic neuropathy. Diabetologia 1983, 24:330-332.

25. Cideciyan AV, Aleman TS, Boye SL, Schwartz SB, Kaushal S, Roman AJ, Pang JJ, Sumaroka A, Windsor EA, Wilson $J \mathrm{M}$, et al: Human gene therapy for RPE65 isomerase deficiency activates the retinoid cycle of vision but with slow rod kinetics. ProcNat/Acad Sci U S A 2008, 105:15112-15117.

26. Cideciyan AV: Leber congenital amaurosis due to RPE65 mutations and its treatment with gene therapy. Prog Retin Eye Res 2010, 29:398-427.

27. Kawasaki A, Munier FL, Leon L, Kardon RH: Pupillometric quantification of residual rod and cone activity in patients with visual loss due to Leber congenital amaurosis [abstract]. Acta Ophthalmol 2011, 89:s248.

28. Zhu D, Moore ST, Raphan T: Robust pupil center detection using a curvature algorithm. Comput Methods Prog Biomed 1999, 59:145-157. 
29. Iskander DR, Collins MJ, Mioschek S, Trunk M: Automatic pupillometry from digital images. IEEE Trans Biomed Eng 2004, 51:1619-1627.

30. Fotiou F, Fountoulakis KN, Goulas A, Alexopoulos L, Palikaras A: Automated standardized pupillometry with optical method for purposes of clinical practice and research. Clin Physiol 2000, 20:336-347.

31. Melillo P, Bracale M, Pecchia L: Nonlinear heart rate variability features for real-life stress detection. Case study: students under stress due to university examination. Biomed Eng Online 2011, 10:96.

32. Melillo P, Fusco R, Sansone M, Bracale M, Pecchia L: Discrimination power of long-term heart rate variability measures for chronic heart failure detection. Med Biol Eng Comput 2011, 49:67-74.

33. Pecchia L, Melillo P, Sansone M, Bracale M: Discrimination power of short-term heart rate variability measures for CHF assessment. IEEE Trans Inf Technol Biomed 2011, 15:40-46.

34. Pecchia L, Melillo P, Bracale M: Remote health monitoring of heart failure with data mining via CART method on HRV features. IEEE Trans Biomed Eng 2011, 58:800-804.

doi:10.1186/1475-925X-11-40

Cite this article as: Melillo et al:: Pupillometric analysis for assessment of gene therapy in Leber Congenital Amaurosis patients. BioMedical Engineering OnLine 2012 11:40.

\section{Submit your next manuscript to BioMed Central and take full advantage of:}

- Convenient online submission

- Thorough peer review

- No space constraints or color figure charges

- Immediate publication on acceptance

- Inclusion in PubMed, CAS, Scopus and Google Scholar

- Research which is freely available for redistribution

Submit your manuscript at www.biomedcentral.com/submit 